\title{
Experiences with HPTN 067/ADAPT Study-Provided Open-Label PrEP Among Women in Cape Town: Facilitators and Barriers Within a Mutuality Framework
}

\author{
K. Rivet Amico ${ }^{1}$ Melissa Wallace ${ }^{2}$ - Linda-Gail Bekker ${ }^{3}$ - Surita Roux ${ }^{2}$. \\ Millicent Atujuna $^{2} \cdot$ Elaine Sebastian $^{2} \cdot$ Bonnie J. Dye $^{4} \cdot$ Vanessa Elharrar $^{5}$. \\ Robert M. Grant ${ }^{6}$
}

Published online: 17 June 2016

(C) The Author(s) 2016. This article is published with open access at Springerlink.com

\begin{abstract}
Placebo-controlled trials of pre-exposure prophylaxis (PrEP) have reported challenges with study-product uptake and use, with the greatest challenges reported in studies with young women in sub-Saharan Africa. We conducted a qualitative sub-study to explore experiences with open-label PrEP among young women in Cape Town, South Africa participating in HTPN 067/Alternative Dosing to Augment Pre-Exposure Prophylaxis Pill Taking (ADAPT). HPTN 067/ADAPT provided open label oral FTC/TDF PrEP to young women in Cape Town, South Africa who were randomized to daily and non-daily PrEP regimens. Following completion of study participation, women were invited into a qualitative sub-study including focus groups and in-depth interviews. Interviews and groups followed a semi-structured guide, were recorded, transcribed, and translated to English from isiXhosa, and coded using framework analysis. Sixty of the 179 women enrolled in HPTN 067/ADAPT participated in either a
\end{abstract}

This work was presented at IAS 2015.

K. Rivet Amico

ramico@umich.edu

1 Department of Health Behavior and Health Education, University of Michigan, Ann Arbor, MI, USA

2 The Desmond Tutu HIV Centre, Cape Town, South Africa

3 Dept of Medicine and Institute of Infectious Disease and Molecular Medicine, The Desmond Tutu HIV Centre, Cape Town, South Africa

4 FHI 360, Durham, NC, USA

5 Clinical Prevention Research Branch, PSP/DAIDS/NIAID/ NIH, Bethesda, MD, USA

6 Gladstone Institutes, San Francisco AIDS Foundation, University of California, San Francisco, CA, USA focus group (six groups for a total of 42 participants) or an in-depth interview $(\mathrm{n}=18)$. This sample of mostly young, unmarried women identified facilitators of and barriers to PrEP use, as well as factors influencing study participation. Cross-cutting themes characterizing discourse suggested that women placed high value on contributing to the wellbeing of one's community (Ubuntu), experienced a degree of skepticism towards PrEP and the study more generally, and reported a wide range of approaches towards PrEP (ranging from active avoidance to high levels of persistence and adherence). A Mutuality Framework is proposed that identifies four dynamics (distrust, uncertainty, alignment, and mutuality) that represent distinct interactions between self, community and study and serve to contextualize women's experiences. Implications for better understanding PrEP use, and non-use, and intervention opportunities are discussed. In this sample of women, PrEP use in the context of an open-label research trial was heavily influenced by underlying beliefs about safety, reciprocity of contributions to community, and trust in transparency and integrity of the research. Greater attention to factors positioning women in the different dynamics of the proposed Mutuality Framework could direct intervention approaches in clinical trials, as well as open-label PrEP scale-up.

Keywords PrEP · Open-label · Women · Adherence . Mutuality framework · Barriers · South Africa

\section{Introduction}

HIV prevention science has produced considerable advances in recent years, with clinical trials demonstrating effectiveness of oral pre-exposure prophylaxis (PrEP) for 
prevention of HIV transmission [1-4]. Results from the iPrEx randomized controlled trial (RCT) [3], Partners PrEP study [1], and CDC's TDF2 study [4] led to US Food and Drug Administration approval of an indication for the first publically available medication to prevent HIV [5]. Recent findings from IPERGAY [6] and PROUD [7], both involving PrEP in cohorts of men who have sex with men (MSM) in France and Canada and the UK, respectively, provide additional support for the effectiveness of PrEP. In contrast, results from the FEM-PrEP study [8] and VOICE [9], both involving women in sub-Saharan Africa, did not demonstrate effectiveness of oral PrEP in the setting of very low rates of PrEP use (cf., [10]). Varying rates of adherence to study product has become a well-recognized threat in PrEP trials and projects [11].

In the wake of the low study-product use observed in studies with sub-Saharan African women, concerns have been raised about the overall acceptability and feasibility of oral PrEP regimens in this population. However, recent qualitative explorations of factors that may have influenced product use in these trials [12-15] suggest that aspects of being in a trial testing an investigational biomedical agent may have heavily influenced product non-use. Feelings towards the research, such as overall support for the project's goals [12] or alternatively ambivalence towards it [15] influenced use and non-use. It is possible that issues inherent in clinical trials with investigational drugs may not generalize to acceptability or feasibility of open-label PrEP. To date, however, experiences among women in subSaharan African women with open-label PrEP, specifically, have not been characterized.

The HPTN 067/ADAPT trial was one of the first open label PrEP studies to be conducted with women in subSaharan Africa [16]. This Phase II, randomized, open-label clinical trial of oral emtricitabine/tenofovir disoproxil fumarate (FTC/TDF) PrEP investigated whether non-daily versus daily regimens resulted in equivalent prophylactic pre- and post-sex coverage. After 6 weeks of once a week directly observed dosing, participants were randomly assigned to one of three unblinded PrEP dosing regimens for 24 weeks of self-administration: daily, twice weekly with a post-sex dose, or event-driven with before and after sex dosing. Only the daily regimen was known to be effective while the study was conducted, and the other two non-daily regimens were presented to participants as investigational.

The aim of HPTN 067/ADAPT was presented to participants as focused on participants' real-world experiences with trying to follow their assigned regimen. Non-adherence was framed as an important and understandable experience and participants were supported with education around their assigned regimen, skills building for dosingschedules and forecasting of sexual events, and motivational support through Next Step Counseling [17] at each study visit. Pills were dispensed from a Wisepill ${ }^{\mathrm{TM}}$ device that recorded each opening and weekly interviews with interviewers tasked solely with collecting pill-taking and sex event data without feedback to study counsellors or clinicians were conducted.

Three sites took part in HPTN 067/ADAPT, including a site in Cape Town, South Africa enrolling heterosexual women, and sites in Bangkok, Thailand and New York City, USA, enrolling men who have sex with men and transgender women. To explore South African women's experiences with open-label PrEP provided within the context of a research study, we conducted a qualitative substudy with women participating in HPTN 067/ADAPT at the Cape Town site. The aim of the qualitative sub study was to provide a nuanced understanding of PrEP use in the context of individual, community, study, and product dynamics. Specifically, we sought to identify global and regimen specific facilitators and barriers to study-provided PrEP use and participation in the open-label PrEP study, and the overarching cross-cutting themes in the narratives that contextualized women's experiences. Similar to previous work in this area [14], we approached the data with the assumption that the levels identified in the socioecological model would generally organize emerging themes. We sought to extend our understanding of these factors by proposing how these levels interact to explain various approaches participants had towards study-provided PrEP across the full range of use and non-use, which reflected initiation or uptake (or avoidance of it), persistence (essentially, adoption of the regimen as something the participant is trying to do), and adherence execution (extent to which the participant is following the dose requirements of the regimen). These approaches to PrEP use emanated from discourse in the current study, but is generally comparable to a recently proposed typology generated from qualitative work in the VOICE study, where patterns of study-product use included non-initiation, discontinuation, mis-implementation, and adherence [18].

In addition to presenting participant reported facilitators and barriers, we identify overarching cross-cutting themes that appeared to characterize aspects of participation and PrEP use that were particularly influential to participant experiences. Drawing from these results, related literature [13-15, 19], and well-vetted social behavioral (socio-ecological [20] and attitude formation [21]) and community (community based participatory research [22]) models, we propose an organizing framework (a Mutuality Framework) to explain different participant approaches to studyprovided PrEP. We propose that a participant's approach to study-provided PrEP is the direct result of a specific, definable interplay between participant, study, and community factors, which depend heavily on one's sense of 
Table 1 Interview guide areas of inquiry for focus groups (FGs) and in-depth-interviews (IDIs)

\begin{tabular}{|c|c|}
\hline Domain & Inquiries/prompts \\
\hline Feasibility/acceptability & Perceptions of feasibility, acceptability and ease of uptake for their assigned regimen \\
\hline Alteration of regimen & Altering the regimen to better 'fit' their daily life or risk behavior \\
\hline Preference for other regimens & $\begin{array}{l}\text { Whether participant(s) would switch to a different regimen if available; what the ideal regimen } \\
\text { would be }\end{array}$ \\
\hline Facilitators and barriers to adherence & Common facilitators and barriers to following assigned regimen \\
\hline Disclosure of participation & Sense of importance that others knew the participant was enrolled in the study \\
\hline $\begin{array}{l}\text { Experiences with participation and study } \\
\text { team }\end{array}$ & $\begin{array}{l}\text { Feelings towards participation, the project, project-staff, and how pill-taking and condom use was } \\
\text { supported }\end{array}$ \\
\hline Recommendations & Recommendations for change in study support or adherence support approach \\
\hline
\end{tabular}

trust in PrEP. The framework encompasses four dynamics labeled by the dominating type of relationship women in that dynamic are anticipated to have with PrEP and the institutions providing it (in the current case, the study): (1) distrust, (2) uncertainty, (3) alignment and (4) mutuality. Intervention strategies targeting enhancing alignment (i.e., positive beliefs in PrEP and goals of the research project) and mutuality (i.e., sense of ownership over and advocacy towards PrEP and goals of the research project) are suggested.

\section{Methods}

From the 179 women randomised to one of the three regimens in the parent study, we planned to recruit a total of 60 participants (34\% of total sample) for participation in either focus group (FG) discussions or in-depth interviews (IDIs), with a similar interview-guide used for FG and IDIs. Per arm, two FGs and six IDIs were planned (for a total of six FGs and 18 IDIs). Sample size was advised by 'information power' [23], where our targeted recruitment reflected the discrete aims of the study and the specificity in inquiry (e.g., use of the same basic semi-structured guide in all FGs and IDIs) and participant groups (e.g., all attended the same clinic, interacted with product, and resided in the same general community). Inclusion criteria for the qualitative sub-study was having finished the on-drug portion of the study (study week 34), while exclusion was having finished over 3-months ago. Convenience sampling was used to identify FG participants, where eligible women were informed of open spots in planned FGs and referred to a coordinator if interested. IDI participants were identified with a combination of convenience and targeted sampling, where attempts were made to include at least two participants from each arm who may have had low adherence based on staff impressions (not Wisepill ${ }^{\mathrm{TM}}$ or drug level testing as that data was not yet available at time of recruitment). Similar to FG recruitment, women were informed of open spots for interviews and scheduled if interested.

FGs and IDIs were conducted in participant preferred language (isiXhosa or English) by an independent experienced interviewer who was not part of the clinical study team and had several years of experience in conducting qualitative interviews with women in the communities surrounding the research site. The development of the interview guide and the approach to analysis of data was situated within a socio-ecological framework [20] and aspects of the information, motivation and behavioral skills model [24] adapted to the current context. The main areas of inquiry from the interview-guide are presented in Table 1.

Data was transcribed and translated into English and analysed using a thematic framework analysis approach [25-27]. Two trained coders sorted transcribed discourse into "frames" determined by interviewer inquiry (which was based on the semi-structured guide). Each frame was then iteratively reviewed for main themes in participant responses to interviewer inquiries. The coding team met throughout this process to review and refine themes, with any disagreements resolved through discussion. Methods were less to ensure interrater reliability [28] than to promote adaptions in the code book that leverage unique insights of coders to create a common, nuanced understanding of frames and themes, which has been evaluated as an approach that produces high level of agreement [29]. Final codes and themes had consensus between coders. Themes in each frame and example quotes supporting the themes were identified, followed by a review and synthesis of all framed content spanning across multiple areas to identify individual, local, cultural or group beliefs and experiences that contextualized women's experiences in the study. Important observations from the qualitative data that were not well captured in the thematic coding from the framework analyses were also evaluated for potential inclusion in cross-cutting themes. These cross-cutting themes advised our formation of a Mutuality Framework 
that sought to explain women's different approaches to study-provided PrEP on the basis of interactions between the participant, the study and study-provided PrEP, and the community. Model development was led by the lead author with iterative vetting with the coding team and the site's community liaisons.

\section{Results}

\section{Participants}

As planned, 60 women participated in the sub-study (42 in focus groups and 18 in interviews). Women were 18-44 years of age (average 26, SD 7), with the majority under the age of 25 . The vast majority (90\%) were not married. Both younger age and being unmarried distinguished the sub-study participants from the study cohort, however the groups were comparable on other demographic or sexual behaviour data.

\section{Themes}

\section{Facilitators of and Barriers to Study-Provided PrEP Use}

As detailed in Table 2, several themes emerged in discourse surrounding facilitators of adherence to the study-provided PrEP pills. This content was organized into the following themes: (1) Efficacy beliefs in PrEP providing effective protection against HIV, (2) perceived HIV-prevention needs/risks highlighted in discourse around enhanced sense of vulnerability to HIV and identifying PrEP use as a source of protection in the event of rape or forced sex, (3) use of concrete adherence strategies such as reminders or pocket dosing, and (4) social support from important significant others that provided concrete help with dose-taking and also removed study-participation disclosure-related barriers. Barriers to adherence, presented in Table 2, included; (1) Attributes of the PrEP pills (e.g., taste and smell) that made dosing unpleasant, (2) perceived side-effects reported largely as nausea and headaches either experienced directly or indirectly through reports of other participants, (3) ARVrelated stigma associated with others assuming the participant is/was HIV-positive because of being seen taking "HIV-medications", and (4) needs for privacy or non-disclosure to important others making dosing more difficult or not possible without risk of undesired disclosure of being part of the study. Specific to non-daily arms, discussion on sex-dependent dosing revealed challenges in predicting sex for pre-sex dosing, but largely centred on difficulty with post-sex dosing because of a perceived mis-match between relaxation or rest following sex and the action-oriented steps needed to take a post-sex dose. Themes and example quotes in Table 2 highlight experiences intentionally limited to facilitators and challenges discussed in relation to dose taking (i.e., regimen execution); other factors that influenced multiple aspects of participation in the study are presented separately below in themes for study participation and engagement more generally. Of note, several of the factors eroding participation in the study noted below have clear implications for also creating challenges to adherence.

\section{Facilitators of and Challenges to Study Participation}

Discourse reflecting reasons for participation, positive or negative consequences of participation, and level of commitment towards and belief in the value of the study and outcomes were reviewed for main themes reflecting facilitators of participation and, conversely, factors challenging participation (Table 3). Participant reflections on facilitators to study engagement were organized into five general themes; (1) Personal experiences with HIV enhancing commitment towards the goals of the study, (2) valuing the package of care received as a participant as unique and beneficial, (3) financial/economic compensation offsetting burden of participation, (4) positive feelings towards the research team, and (5) commitment to HIV prevention research as a benefit to one's community. Discourse highlighting potential factors negatively impacting full participation or engagement in the study included; (1) Concerns about safety of PrEP and confluence of directly or indirectly experienced side-effects exacerbating these concerns, (2) community distrust of study and/or PrEP and women's participation in the study expressed as beliefs women were getting treatment for HIV, the clinic was selling blood collected from participants, and devaluation of participants as only interested in money, not community prevention, and (3) negative clinic experiences largely involving discomfort with sexual behaviour questions and a lack of transparency in what was being done with that information, as well as feeling wrongly accused of nonadherence. As indicated in the sample quotes in Table 3, women discussed both sources of pride in being an active participant as well as considerable "cost" in terms of negative pressure from community members and important others. This tension was evidenced across discourse, leading to the further identification of overarching crosscutting themes that better captured potential drivers, responses and management of these tensions.

\section{Cross-Cutting Themes/Narratives Contextualizing Approach to Study-Provided PrEP}

Several underlying "cross-cutting" themes were identified. Two reflected cultural contextual factors that likely influenced women's overall experiences in the study and with 
Table 2 Facilitators of and barriers to study-provided PrEP use

\begin{tabular}{lll}
\hline Theme & Defined as discourse on... & Example quotes \\
\hline
\end{tabular}

Facilitators of PrEP use

Efficacy beliefs

Beliefs that PrEP works to prevent HIV

Perceived HIV-

prevention needs/risks

Use of concrete

adherence strategies

Social support for use

Support from partner/friend/family for taking PrEP protect HIV-negative status; discussion of prevention in context of rape/forced sex

Strategies used for adherence$$
\text { taking PrEP }
$$

\section{Barriers to PrEP use \\ Attributes of PrEP pills (taste, smell)}

Side-effects attributed to PrEP

ARV-related stigma

Needs for privacy/nondisclosure

Negative perceptions of pill attributes Negative physical experiences attributed
to using PrEP in self or others

Fears that PrEP use will be misattributed to HIV-treatment; participant will be assumed to be HIV-positive
Non-disclosure of study participation to significant others, due to anticipated stigma, misunderstanding or lack of support
'What motivated me is the fact that they protect me from getting HIV, because sometimes I forget to use a condom with my boyfriend that is why I continued using the pills. I had that hope that the pills will protect me..." D IDI

"The treatment made me safe so I continued taking the pills." E IDI

"I heard here at the site that these pills work and that they were being tested overseas too and that the results proved that these pills do work so that made me take the pills." E FG

"As I said before, it made me want to protect myself. Before I was involved in the study, I didn't care as much as I do now." T IDI

“... I also knew that this pill will help me in any case like if I was to be raped I would not be infected with HIV" D IDI

"I didn't set my phone or anything like that. I knew that if Generations [a popular television series] is about to begin, I would take my pill." D FG

"I would keep the tablets in my pocket so that I always remember to drink the tablets" E IDI

"My friends would also help me because they knew at a certain time I was supposed to take the pill. So it was those kinds of things that helped me." T FG

"The boyfriend that I was staying with was very supportive and he always encourages me to drink the tablets." E IDI

"Yes, at the beginning I was asking myself, how am I going to be able to swallow this big pill and as time goes on, I was able to swallow them." D IDI

"What I found difficult was the way it smelled, it made me nauseous. So when the time came for me to take it, I had to think hard about it. I wasn't too happy taking it." D FG

"At first it was hard because they were not good for my immune system but they have told me here that at first I might have some side effects such as always feel[ing] hungry, dizziness and they made me to have a small rush but as time goes on, I got used to it." D IDI

"Plus negative response from friends ... they compare Truvada ${ }^{\circledR}$ to ARVs because they know someone who was taking the same medication and ended up being HIV positive." D IDI

"We are very shy of walking around with pills in our bags, because we are scared of what people would say, because let us say you take out your pills and take them at the party, some people won't even ask- they will just say it's an ARV." E FG

"The problem was that I didn't tell my boyfriend that I was taking the Truvada ${ }^{\circledR}$. So when I went to his place, I wouldn't take it along" T FG

"So you are now sitting with friends and you see that the time is about to arrive. So what will they say if I were to take these pills in front of them? My friends are going to judge me. So I end up not taking them then." E FG 
Table 2 continued

\begin{tabular}{|c|c|c|}
\hline Theme & Defined as discourse on... & Example quotes \\
\hline \multicolumn{3}{|l|}{ Non-daily regimens } \\
\hline \multirow[t]{4}{*}{ Sex-dependent doses } & $\begin{array}{l}\text { Difficulty in determining whether or not } \\
\text { sex would occur (for pre-sex dosing) } \\
\text { and a mismatch between PrEP dosing } \\
\text { and the post-sex milieu }\end{array}$ & $\begin{array}{l}\text { "What would get me to forget is that-I live with my } \\
\text { boyfriend, right, okay. So maybe we're lying on the bed } \\
\text { together and then sex just happens... Now my pills sit in a } \\
\text { divider and sometimes they are looking at me, but I am busy } \\
\text { at the moment... So I will have sex and then will wait for the } \\
\text { appropriate time for me to take the after sex pills." E FG }\end{array}$ \\
\hline & & $\begin{array}{l}\text { "The regimen that we were in was very difficult. Let's say that } \\
\text { you are in town and your partner phones you and says: } \\
\text { "Baby, please come this way when you're finished in town." } \\
\text { Now you might not have a chance to stop off at home } \\
\text { because it could be late." E FG }\end{array}$ \\
\hline & & $\begin{array}{l}\text { "And sometimes, after sex, you want to sleep. Maybe you're } \\
\text { tired. You don't think about taking pills. Maybe you guys are } \\
\text { sitting together and talking since you don't see each other so } \\
\text { often. So then you will forget the pills." E IDI }\end{array}$ \\
\hline & & $\begin{array}{l}\text { "After sex.... After I have just finished having sex, it's nice to } \\
\text { sit back and relax a bit." T FG }\end{array}$ \\
\hline
\end{tabular}

$D$ daily regimen, $T$ time-driven regimen, $E$ event-driven regimen, $I D I$ in depth interview participant, $F G$ focus group participant

PrEP: (1) A prioritization of contributions to the community, which is consistent with a South African worldview Ubuntu, and (2) a pervasive skepticism from both participants and communities about the trial, the product, and procedures. The final theme also spanned across discourse but was more reflective of ways in which women used and did not use PrEP: (3) variability in approaches to regimen uptake, persistence and adherence, between participants and over time. Each cross-cutting theme is discussed in detail below, and our integration of these themes into a more comprehensive framework for understanding the experiences of women in the study with hypotheses concerning how factors at the participant, study and community level interacted is provided through a proposed Mutuality Framework.

Ubuntu Discourse about the personal protective value of PrEP was present but the desire to contribute to something good to the community was resounding. Note that this differed from altruism in that participants reflected on wanting efforts to be valued in their community specifically, highlighting strong reciprocity desires. Prioritization of community well-being and feeling aligned with the "good" of one's society is highly consistent with the concept of Ubuntu. Ubuntu as a worldview emerged in the mid-19th century to describe South African communities working together as communities, and identifies that 'humanity' exists in the interactions of groups of individuals. It reflects beliefs that society, over individuals, gives meaning and relevance, emphasizes collective responsibility, and commitment towards health and well-being of one's community.
Skepticism Narratives reflected multiple experiences that spoke to an underlying skepticism towards study-provided PrEP and research more generally. This could be considered "healthy" skepticism in the sense that the cultural, social and political history in communities participating in this study have very recent and on-going experiences with oppression and discrimination that promote skepticism as an important safety precaution. Ongoing economic disparities and poverty characterize daily life for most participants. Medical establishments and biomedical research centers, even those experienced as providing valuable contributions to the participants, can be affiliated with majority group(s) who are seen as responsible for past injustices and ongoing disparities, or overt negligence towards the safety and rights of members of the community. It is important to note that skepticism as used here is not an outright rejection of the study, but rather an approach to the study that seeks "proof" to build trust or to confirm that the study and products should not be trusted. Importantly, high quality interactions with the study site and staff are not sufficient to overcome negative expectations that are deeply seeded in the history of medical services.

Variable Approaches to Study-Provided PrEP How participants approached study-provided PrEP varied considerably, from active avoidance of taking doses and disclosure of such to the study team, to strong commitments to use PrEP and advocacy in support of the study and PrEP in the community and with other participants (PrEP champions). Several women discussed avoiding PrEP use entirely, and there was ample discourse about "other" 
Table 3 Facilitators of and challenges to study participation

\begin{tabular}{lll}
\hline Theme & Defined as discourse on... & Example quotes
\end{tabular}

Facilitators of study participation

Personal experiences with HIV

Valuing the package of care received as a participant

Financial/economic compensation

Positive feelings towards the research team

Commitment to HIV prevention research

Challenges to participation

Concerns about safety

Community distrust of study and/or PrEP and women's participation in the study

Negative clinic experiences
Desires to contribute towards HIV prevention because of negative impact of HIV on family, friends, or community

The unique benefits of being in the study in terms of the medical care and screening not easily available outside of the study

Reimbursements as motivating participation in the study

Experiences, beliefs or feelings towards study team that were positive or motivating

Discourse of a shared vision with the study in terms of working together to make real contributions to HIV prevention in their community

Study provided PrEP as unsafe or less safe than informed by the research team

Community rumors/convictions that women would get HIV through participation, have HIV, or prioritize themselves and receiving money for participation over the community

Experiences at clinic site that were negative or considered burdensome; feelings of lack of transparency/ feeling accused
"I joined because I have a family member who passed on because of HIV, so I decided to take part because I will also benefit". E FG

"You know, when we're in the township, it can be difficult for us to go test at the clinic and you won't know what your status is. So at least when you come here, you can find out whether you are sick or not. So that supported me because I got to know about my health." E IDI

"Maybe you just want to be cautious about your health because here at the study they look at a lot of things you don't drink the pills only. That is what I liked" D IDI

"They [other participants] also told me about the difficulties they had but then they endured them. Another one told that she is enduring them because you get money in this study, like a lot of money." T IDI

"It's the way they treat us here at [site name]. It's the way the counselors also speak to us. They help you understand the way in which these pills are meant to be taken. They don't force you." T IDI

"All the staff members were supportive I enjoy coming here." E IDI

"What made it easy for me was that it's helping the community. It's not only helping me. So I am happy that there were people who were supporting me." D FG

"I was following the instructions and I told myself that I was doing it for a purpose. ...to check as to whether this research works for other people." D IDI

"People were not drinking the tablets because they were flushing them down toilets because they were [...] experiencing side effects like headaches, stomach ache and gaining weight." E IDI

“...I was scared of getting side effects hence I would throw the pills away...." T IDI

"I was okay but got worried because people were talking about side effects." T IDI

"Yoh! People say that they give you AIDS there!" E FG

“... and my friend also said I am looking for trouble by joining this study she had this whole idea of how I could catch HIV." T FG

"And as for my friends... they were telling me that I am only carrying on with this study because I wanted money" D IDI

"My family never encouraged me, especially my sister. She just told me that I was going to get AIDS. She said: 'They take your blood and sell it." " D FG

"They irritated me because the same question is asked every day: "address, contacts, phone numbers" - all the time... He would ask the same questions. ... Then when you come back you have to explain again." T FG

“... and traditionally for us black people we don't disclose info like that easily to anyone, it's embarrassing and especially when they ask these unexpected questions." $\mathrm{T}$ FG

“...it was all just irritating, and they would look you in the face plus they wouldn't say if you right or wrong, they would just write down what you saying." T FG

"...the counselors were telling us that we are throwing the pills away, which it was not all of us." T IDI

$D$ daily regimen, $T$ time-driven regimen, $E$ event-driven regimen, $I D I$ in depth interview participant, $F G$ focus group participant 


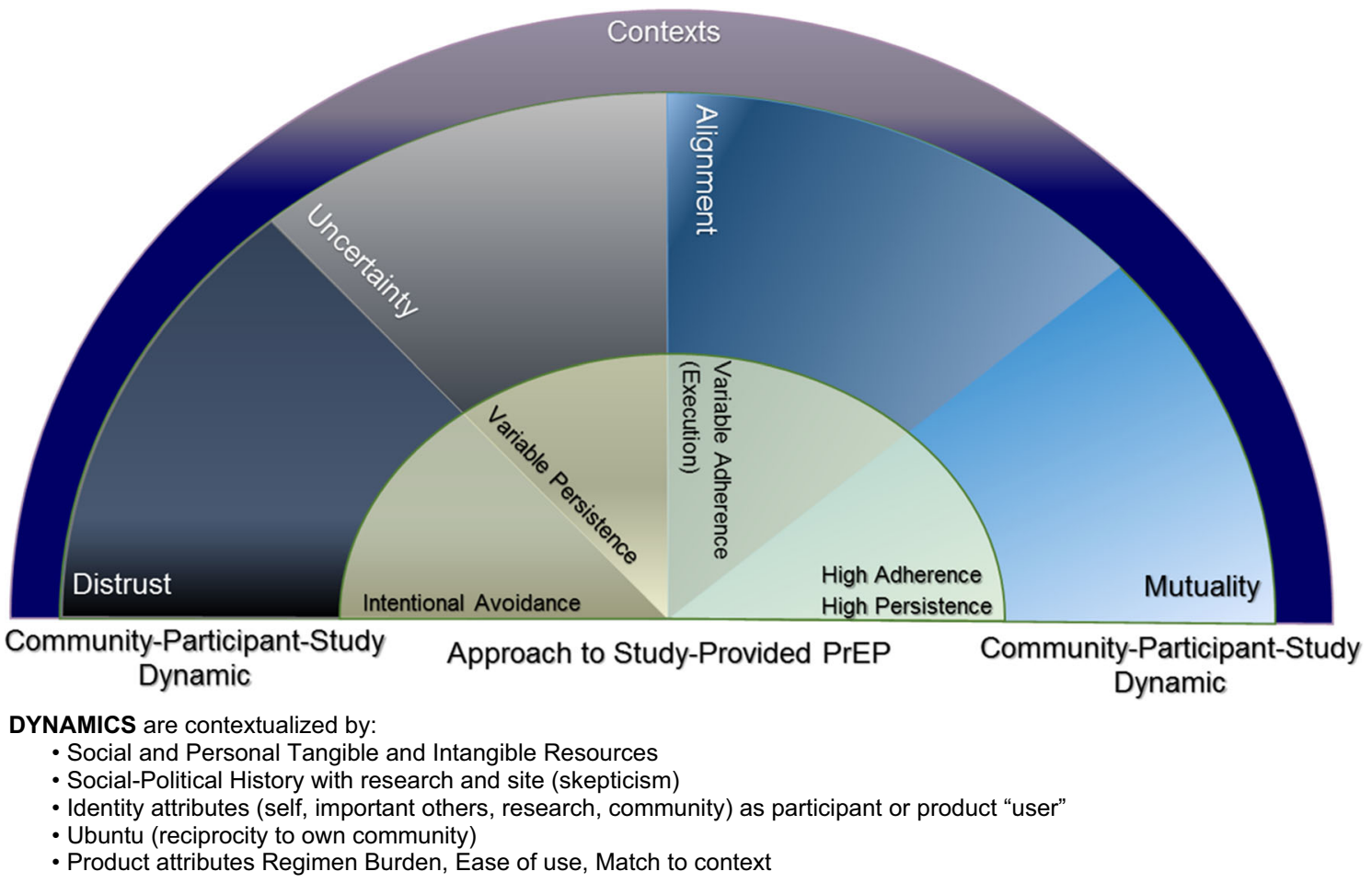

Fig. 1 Mutuality framework

participants discarding drug, opening and closing the electronic drug monitoring device (Wisepill ${ }^{\mathrm{TM}}$ ) to appear adherent, and advising other participants to avoid PrEP across study arms. However, there was also considerable discussion about actively engaging other participants and challenging negative stereotypes of participants and skepticism in the community. In between the extremes of intentional avoidance and 'champions', women discussed variability in persistence (defined here as periods of consistent engagement with the regimen or commitment to trying to adhere) and execution of regimen (adherence towards a regimen one is trying to take).

\section{Synthesis of Findings: A Mutuality Framework}

The cross-cutting and specific themes were used to develop a framework to understand interactions between participant, community and study and how these influenced women's approaches to study-provided PrEP. Throughout discourse a tension in negotiating dynamics between self, study and community was clear. We characterize approaches to study-provided PrEP as ranging from intentional avoidance of PrEP dosing to strong persistence and adherence. These approaches are situated within larger social-cultural and resource contexts including; the value of social and personal resources afforded through participation, the social-political community history with biomedical research and medical institutions, identity attributes (how participant and PrEP user is characterized internally, to important others, in the community), cultural world view emphasizing reciprocity to one's community, and product attributes and regimen burden or ease of use. As indicated in Fig. 1, these "context" factors apply to the formation, maintenance and/or movement between the dynamics.

We adopt the term 'dynamics' to refer to the constellation of factors influencing women's experiences with study-provided PrEP. These are not intended to characterize people, rather they are ways of thinking about the participant's approach to study-provided PrEP at a given point in time as the result of her negotiation of tensions and synergies between herself, the study and her community. Table 4 presents each dynamic in terms of characterization, approach to study-provided PrEP, drivers of that approach, and implications for intervention. The Mutuality Framework (Fig. 1) and each dynamic detailed below represent our integration of the narratives shared by women in the study, while also expanding beyond the discourse to incorporate findings in previous literature, multiple models and theories pertinent to participatory research, socioecological and social-determinants frameworks, and cultural models. As such, the Mutuality Framework we present is advised by factors identified in the current research but extends beyond discourse to propose a new model 
Table 4 Dynamics in Mutuality Framework

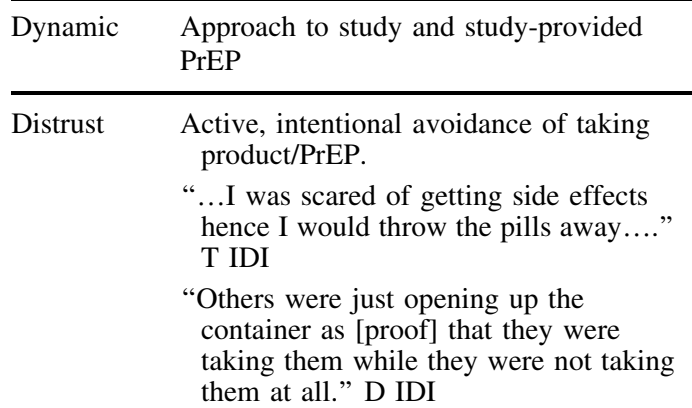

them at all." D IDI
Uncertainty Variable persistence with study-provided PrEP- on-again/off-again engagement with trying to use study-provided PrEP.

"Firstly, people say that we're risking our lives by getting involved in HIV research." E FG

"But then I ended up thinking and thinking and thinking about this, whether there really isn't anything [HIV] they are giving us here." D IDI

Caused by...

Intervention implications and possible strategies

Rejection of integrity of study (goals, potential reciprocity to community) and safety of products/PrEP and efficacy.
Strategies targeting changing beliefs in safety, reciprocity, and efficacy of product or integrity/relevance of research findings (for efficacy trials)

Possible change strategies:

Community theatre with roles for "pro" and "cons" of drug safety or study integrity where turns are taken in giving voice to each "side", ending with thoughts on what evidence/experiences would convince one side or the other

Normalization of skepticism and overt discussion of pros/cons allowing for exploration of each

Community engagement and mobilization events (i.e., CBPR strategies)

Designs and programs that allow for discontinuation or not using PrEP while remaining in cohort

Creating "task force" teams of participants who are tasked with and resourced to perform fact-finding missions about study and/or products

Skeptical exploration of whether or not to trust study, PrEP, or providers of PrEP (the research study, demonstration project, or health agency)

In addition to changing beliefs (above), strategies targeting enhancing beliefs of safety, reciprocity, and efficacy

Possible change strategies:

PrEP study or program awareness campaigns that invite open discussion of potential medical mistrust from social-historical and political perspectives

Promote exploration of ambivalence as reasonable and valid with a focus on identifying what "data" would be needed to assure participant

Adopt high transparency strategies that explain aspects of procedures, protocol or PrEP programs that are uncommon in communities- for example, media providing "proof" of legitimacy of tests, samples or monitoring (video of blood collection, where it is shipped with pictures of labs, and disposal after processing)

Engage peers, champions, and trusted individuals to lead debates and discussions about integrity, truth, and reciprocity

If the PrEP agent is known to be effective, emphasize this aspect of potential value through multiple modalities (pictures, media, theatre and other methods of depicting efficacy)

Create opportunities to build sense of ownership in trial- task participants with conducting evaluations of experiences at clinic, quality of care received, and social harms (negative experiences) in community that are fed back to the research team and acted upon 
Table 4 continued

\begin{tabular}{|c|c|c|c|}
\hline Dynamic & $\begin{array}{l}\text { Approach to study and study-provided } \\
\text { PrEP }\end{array}$ & Caused by... & $\begin{array}{l}\text { Intervention implications and possible } \\
\text { strategies }\end{array}$ \\
\hline Alignment & $\begin{array}{l}\text { Whereas persistence (trying to take } \\
\text { study-provided PrEP) is likely good; } \\
\text { execution adherence is anticipated to } \\
\text { vary on the basis of adherence skills } \\
\text { (strategies) and degree of positive } \\
\text { beliefs about value of PrEP and } \\
\text { adherence } \\
\text { "... I wouldn't do any of that [not take } \\
\text { the tablets] because I want to see if } \\
\text { these pills really, really work" T FG } \\
\text { "I also wanted to continue taking it to the } \\
\text { end and if I hear that the pill did its job } \\
\text { and helped people, I will be proud of } \\
\text { that." E FG }\end{array}$ & $\begin{array}{l}\text { Provisional acceptance that the study and } \\
\text { products provided by study do benefit } \\
\text { self and community in ways that are } \\
\text { relevant and meaningful }\end{array}$ & $\begin{array}{l}\text { Support should target maintaining trust in } \\
\text { study and positive beliefs about study- } \\
\text { provided PrEP use and optimizing } \\
\text { adherence. } \\
\text { Possible change strategies: } \\
\text { Barriers based discussions to identify } \\
\text { adherence challenges and resources and } \\
\text { skills that could be used to address them } \\
\text { Peer based support for adherence and } \\
\text { developing strategies to promote } \\
\text { adherence } \\
\text { Real-time monitoring may help to } \\
\text { provide reminders and problem solving } \\
\text { support as and when needed } \\
\text { Exploration of collected dosing data (as } \\
\text { available) to identify strengths, barriers } \\
\text { and potential strategies }\end{array}$ \\
\hline Mutuality & $\begin{array}{l}\text { Both persistence and execution adherence } \\
\text { are generally high/good } \\
\text { “... and I said: "Look here, ask me. And } \\
\text { don't you dare say I have HIV, telling } \\
\text { everyone in this shop. We are doing } \\
\text { research here... to see whether the pills } \\
\text { can protect someone from HIV.” E FG } \\
\text { “... [people] in the study must help them. } \\
\text { They must be proud to talk about the } \\
\text { pills and encourage other people.” T } \\
\text { FG }\end{array}$ & $\begin{array}{l}\text { Ownership of PrEP and/or goals of the } \\
\text { study or program to the point of } \\
\text { advocacy }\end{array}$ & $\begin{array}{l}\text { Support for uptake, persistence and } \\
\text { adherence are not generally needed in } \\
\text { this dynamic. Rather, avoiding eroding } \\
\text { mutuality is essential and developing } \\
\text { avenues for collaboration offers } \\
\text { opportunities to mobilize participant } \\
\text { groups and communities } \\
\text { Possible strategies to retain women in this } \\
\text { dynamic: } \\
\text { Create programs for peer mentors, } \\
\text { community champions, and other roles } \\
\text { that facilitate advocacy } \\
\text { Create sister-groups where women can } \\
\text { lead discussions among women in the } \\
\text { study or program } \\
\text { Create and use rotating participant } \\
\text { advisory panels where women can take } \\
\text { on valued leadership positions within } \\
\text { the study or program } \\
\text { Engage women in the development of a } \\
\text { plan for how results of the study or } \\
\text { program will be disseminated to } \\
\text { community and policy makers } \\
\text { Facilitate the creation of advocacy groups } \\
\text { that can lead local and regional efforts } \\
\text { to enhance awareness in communities } \\
\text { and represent community with local } \\
\text { health ministries, feeding back to } \\
\text { community progress and reasons for } \\
\text { delays in rolling out diverse prevention } \\
\text { strategies }\end{array}$ \\
\hline
\end{tabular}

offering a more complete, contextually-grounded, theoretical conceptualization of experiences with biomedical HIV-prevention.

\section{Dynamic 1: Distrust}

In the distrust dynamic one is anticipated to avoid use of study-provided PrEP because of beliefs that the pills provided are unsafe and there is uncertainty about the protection of participants in the study and limited expectations of community or personal benefit from the perceived high-risk of using study-provided PrEP. The aspect of adherence most impacted in this dynamic is uptake. Normative beliefs center on other participants also avoiding use of the pills, and use of the pills reflecting naiveté in other participants. Women in this dynamic attempt to protect other participants by encouraging non-use and drawing from examples of experiencing side-effects and 
sero-conversions as proof of conviction that participants are at risk. Efforts from the study team to debunk 'misinformation' or rumors that minimize or fail to recognize the legitimacy of concerns are expected to reinforce distrust rather than reduce it. Women in this dynamic are expected to avoid open discussions with the study team about non-use of pills, or appearing non-adherent on selfreport or other measures that can be adjusted (e.g., announced pill-counts). Arguably, women experiencing distrust are likely the most difficult to work with from a study team perspective because their lack of trust in the integrity and transparency of the study limit open discourse. They may, however, be identified through a lack of drug concentration in combination with reports of high adherence, which could open a window for discourse. Studies that allow for participation without PrEP use (e.g., a no-PrEP arm) may be better positioned to decrease this dynamic or offer opportunities to women to come off PrEP while they consider or re-consider safety. We hypothesize that the presence of this dynamic is likely in contexts where there are driving structural or economic motivators to participate- strong enough to persuade a woman who is experiencing high levels of distrust and fear associated with taking PrEP to nonetheless enroll and show up for visits and procedures. In contexts where participation in the study does not afford high-value, unique benefits, individuals experiencing distrust would not likely enroll or be retained in the study. Interventions to promote movement out of the distrust dynamic may include community based participatory research practices to reduce some of the factors driving the distrust dynamic, and any strategy that dismantles beliefs about conspiracy, hidden risks, or disregard for safety. Strategies that provide opportunities for participants or patients to take on active roles in monitoring quality of service delivery may similarly work to influence beliefs in the integrity and transparency of programs.

\section{Dynamic 2: Uncertainty}

Individuals in this dynamic are expected to oscillate between PrEP use and non-use in response to shifts between feeling that PrEP use is safe and accurately represented by the study and feeling that PrEP use is unsafe and that the study mischaracterizes risks involved. The aspect of adherence most influenced in this dynamic is hypothesized to be persistence- as periods of attempting to follow the PrEP regimen is interspersed with periods of avoiding it. Objective measures of drug concentrations may mischaracterize individuals in this dynamic if the window is too short (e.g., dosing in last 3 days or last week) and per-week dosing may be a poor characterization as women would be expected to have some weeks on PrEP and some weeks off of it. Electronic dose monitoring devices may have utility to the extent that they are not overtly manipulated to appear persistent even when not taking PrEP. Women's experiences in this dynamic are characterized by feeling pulled in different directions and the internal debate over whether or not the pills and the study more generally can be trusted is influenced by ongoing experiences with the pills (e.g., side-effects), study team (e.g., positive and negative experiences with study team members), other participants (e.g., appeals from other participants to trust or reject the study and, relatedly, PrEP), important others (e.g., positive and negative influence of family members and partners), and community (e.g., hearing rumors or being ascribed negative traits ['selling out' community for money] or positive ones from others in the community). Normative beliefs of what other participants are doing with their pills are fluid and not crystalized as definitely dumping or definitely taking the pills, while hearing of other women's experiences is anticipated to be particularly impactful in moving out of this dynamic. We conjecture that the experience of this dynamic is tense and uncomfortable, which resolves only when beliefs shift towards either distrust or stronger alignment with the study. It is unclear how typical education and counseling on adherence may play a part in this dynamic, as it could be argued that clear information and support from the study team could move the participant closer towards the aims of the study. Alternatively, if information appears one-sided (reasons why one should or must use PrEP) or dismissive (stating PrEP is safe without further exploration), it could propel rather than diminish concerns. Adherence counseling focused on identification and remediation of barriers to dosing assumes a shared interest in high adherence, which is likely mismatched for those in the uncertainty dynamic where the participant is still considering her willingness to try a regimen. Like the distrust dynamic, Community Based Participatory Research (CBPR) strategies, engaging individuals in service delivery monitoring, and designs or programs that allow for non-use of PrEP may be helpful. Other strategies that promote discussions of uncertainty and ambiguity focused on decision making around uptake and persistence, preferably with the support of trusted individuals (peers or participant "champions"), may be promising.

\section{Dynamic 3: Alignment}

Individuals in this dynamic are anticipated to be generally engaged with trying to use PrEP, meaning they are likely persistent but may have challenges to consistent dosing due to commonly reported factors such as mustering motivation to dose in specific situations, remembering, negotiating privacy, or having doses accessible. Pill-use is characterized as persistent but with varying levels of adherence. The 
study, procedures and the pills are generally seen as safe and the goals of the study are generally considered trustworthy, with potential to benefit one's self and one's community. The balance of risk and reward is one of minimal risk and possible benefit. Positive beliefs are strong enough to build resilience to negative community pressure, although actively shifting community beliefs is not a priority. Normative beliefs about what other participants are doing, or not doing, exert less of an impact, and one's own experiences with PrEP, the study and significant others are more influential. Women in this dynamic attempt to follow recommendations and regimens, and have more resilience in reporting non-adherence back to study team members. A diverse set of strategies may be helpful for women in this dynamic. Strategies using objective markers of PrEP use may assist women in identifying patterns that produce optimal and sub-optimal levels of protection. Of note, the education and counseling offered in many studies and programs that focus on unpacking potential facilitators and barriers to adherence and building skills are likely to be most appropriate for individuals in this dynamic, as open discourse is possible and there is a shared goal of adherence.

\section{Dynamic 4: Mutuality}

Individuals in this dynamic have a high degree of ownership over PrEP and/or the goals of the study. Persistence and adherence are both anticipated to be high and consistent largely due to strong positive beliefs in PrEP and the study's ability to make lasting, real contributions to personal and community health and wellness. In this dynamic, women are likely aware of participants in other dynamics (particularly rejection and uncertainty) and community concerns about the study or women participating in it. They appreciate that normative beliefs for PrEP (and biomedical prevention more generally) are diverse and fragmented in the community. Unique to this dynamic is the response women have to these experiences. They respond by overtly challenging the beliefs of others, 'vouching' for the integrity of the study and the product(s), and seeking out opportunities in the community to shift beliefs. Their advocacy positions them as "PrEP Champions" in both the community outside of the study and within the study itself. Their accumulated experiences with PrEP can position them as more expert in terms of adherence than the study team, who typically do not use PrEP or have lived experience with taking it. It is not clear that women in this dynamic need study-provided support for persistence or adherence, aside from being responsive to specific questions or issues raised by the participant. It may be more important to avoid the introduction of experiences that may move someone out of the mutuality dynamic, and create new opportunities to allow for thus dynamic in the context of research trials. Asking women in this dynamic to reflect on doses missed (self-reported or objectively monitored) may hold appeal to them if presented as for research or data tracking purposes. However, if framed as for their own benefit, women may feel such conversations with staff or study team members, who themselves have little lived experience with taking PrEP and advocating for it in their communities, belittling or dismissive of their own expertise. Support for adherence may be best positioned as "as needed" for women in this dynamic. Other activities, however, could help to keep women in this dynamic and moreover could engage these women in assisting others. Creating opportunities to serve as peer or participant champions who could support other participants, speak at community events, provide input into policy forums, or advise the study team on recommendations for working effectively with community and participants in other dynamics would likely be more appropriate than a focus exclusively on adherence.

\section{Discussion}

Discourse from predominantly young, unmarried women who participated in the HPTN 067/ADAPT trial suggested that approaches to open-label PrEP provided as daily, twice-weekly plus post-sex dose, or pre- and post-sex dosing varied, although many women spoke of high commitment, persistence and adherence to their regimen. Facilitators and barriers identified to dosing were generally consistent with the literature on adherence to antiretroviral therapy (ART) [30], prevention medications (e.g., hormonal contraception [31]), post-sex dosing challenges [32], as well as recent evaluations of study-product use in FEMPrEP and VOICE [12-15]. In our sample of women, specific challenges to non-daily dosing appeared centered on the context in which participants had sex (e.g., unplanned, as available and typically outside of one's home) and the context surrounding post-sex (e.g., where relaxation takes precedence over action-oriented prevention behaviors such as dosing). Moreover, throughout the discourse, women reflected on negotiating the potential use of study-provided PrEP in a context where there were substantial concerns about safety and integrity of the trial and procedures, in many cases not knowing whether or not the PrEP provided could be trusted or the study would indeed benefit one's community.

From the narratives collected, we constructed a Mutuality Framework which proposes a characterization of how the intersections, or dynamics, between self, community and study impacted overall approaches to study-provided PrEP. We propose diverse sets of strategies that could be 
implemented by study teams and/or PrEP implementation programs as ways to enhance movement towards alignment. Typical adherence support offered within trials and implementation programs that seek to optimize execution adherence (doses taken as recommended) is well-matched to the alignment dynamic, but may be poorly matched in the remaining three dynamics (distrust, uncertainty, or mutuality). Future research should target the evaluation of CBPR strategies [33] and Good Participatory Practices [34], as well as other innovative approaches, in shifting levels of trust in both biomedical agents and biomedical research. Efforts to engage social behavioural research to measure dynamics that will influence PrEP uptake, through the development of new or adaptation of existing scales (i.e., the Group Based Medical Mistrust Scale [35, 36]), characterising movement through dynamics over time and in response to events and experiences, and strategies to effectively shift distributions in our framework are important next steps.

Although our results are specific to the group of participants engaged in this study and may not characterize experiences of participants not included in the interviews or focus groups, we do believe that the overall framework we developed is generalizable. We cannot, however, presently speak to whether or not the data collected would have differed considerably if the only regimen examined in HPTN 067/ADAPT was a proven one. Having other 'investigational regimens', even if the drug itself was open label, may have created challenges to perceived safety and clearly could have challenged feelings of efficacy. Even in light of this, we do believe the Mutuality Framework may apply to other projects and PrEP roll-out in areas where there may be skepticism towards biomedical prevention, PrEP specifically, or the agencies that provide it. Moreover, our framework is highly compatible with models of innovation adoption [37], as well as process models for behaviour adoption [38] and participatory engagement models (cf., [39]), suggesting some applicability to PrEP use more generally. However, future research is needed to evaluate the replication and applicability of the dynamics to "real-world" PrEP use.

The critical role of "medical mistrust" in treatment adherence and research participation is well-established, has clear roots in patterns of discrimination and promotes widespread health disparities [40-43]. Our results add to this literature by offering a nuanced framework for understanding the manner in these factors may play out in biomedical prevention studies. We believe many of the core drivers we identify and manner in which systems interact are generalizable to outside of a research trial. Implications to PrEP roll-out may include careful attention to the distribution of the Mutuality Framework dynamics in targeted communities, and how planned dissemination of PrEP may foster or mitigate skepticism, doubt, and distrust.
For example, when PrEP demonstration projects or PrEPspecific clinics offer care beyond that which is available in the community, larger distributions in the distrust and uncertainty dynamics would be expected. Education and awareness activities with trusted sources (peers, community members, traditional healers) in trusted venues (faithbased venues, community organizations) would be expected to speak to those experiencing distrust and uncertainty, while scientific experts at town-halls, speaking events and policy forums may speak more to those in alignment and mutuality dynamics. Promoting and capacitating those in mutuality to lead community campaigns and engage policy-makers directly may have high impact.

In summary, in combination with results from the main HPTN 067/ADAPT study [16] in Cape Town, women in sub-Saharan Africa found PrEP feasible and promising as a self-directed tool for HIV-prevention. Women approached PrEP in different ways, which we believe was dependent on levels of perceived safety, trust in PrEP and those providing it, and investments in protecting one's community either from PrEP or with it. Past literature has clearly documented the need for effective, generalizable interventions to promote high levels of collaboration and trust in communities for biomedical intervention, prevention, and treatment $[42,44]$. As PrEP implementation programs unfold around the world, there is a real urgency to identify how to "get it right". As suggested by our results, there are many avenues to consider for how to potentially engage communities around PrEP. Importantly, should our Mutuality Framework offer a replicable, generalizable depiction of experiences with PrEP, there are also avenues that could distance communities and create substantial long-lasting barriers in the adoption of biomedical HIV-prevention innovations. Appreciating the cultural, political and historical factors contextualizing PrEP and other biomedical prevention strategies will be a critical ingredient in successful implementation programs.

Acknowledgments HIV Prevention Trials Network is sponsored by the U.S. National Institute of Allergy and Infectious Diseases, the U.S. National Institute of Mental Health, and the U.S. National Institute on Drug Abuse, all components of the U.S. National Institutes of Health. This work would not have been possible without contributions from HIV Prevention Trials Network support from FHI 360, the research team at the Emavundleni Research Centre in Cape Town, the full study team, and all of the participants who shared their experiences. Additionally, we wish to thank Kate Snyder for her contributions coding the data.

Funding This work was funded by the HPTN network and Gladstone Institute of Virology.

\section{Compliance With Ethical Standards}

Conflicts of interest Gilead Sciences provided study drug for the parent project (HPTN 067/the ADAPT study) and to the Desmond 
Tutu Health Foundation (DTHF). K Rivet Amico has an unrestricted educational grant from Gilead Sciences through the University of Michigan. Melissa Wallace, Linda-Gail Bekker, Surita Roux, Millicent Atujuna, Elaine Sebastian, Bonnie J. Dye, Vanessa Elharrar, and Robert M. Grant declare no conflict of interest.

Ethical approval All procedures performed in studies involving human participants were in accordance with the ethical standards of the institutional and/or national research committee and with the 1964 Helsinki declaration and its later amendments or comparable ethical standards.

Open Access This article is distributed under the terms of the Creative Commons Attribution 4.0 International License (http://crea tivecommons.org/licenses/by/4.0/), which permits unrestricted use, distribution, and reproduction in any medium, provided you give appropriate credit to the original author(s) and the source, provide a link to the Creative Commons license, and indicate if changes were made.

\section{References}

1. Baeten JM, Donnell D, Ndase P, Mugo NR, Campbell JD, Wangisi J, et al. Antiretroviral prophylaxis for HIV prevention in heterosexual men and women. N Engl J Med. 2012;367:399-410.

2. Choopanya K, Martin M, Suntharasamai P, Sangkum U, Mock PA, Leethochawalit M, et al. Antiretroviral prophylaxis for HIV infection in injecting drug users in Bangkok, Thailand (the Bangkok Tenofovir Study): a randomised, double-blind, placebocontrolled phase 3 trial. Lancet. 2013;381:2083-90.

3. Grant RM, Lama JR, Anderson PL, McMahan V, Liu AY, Vargas L, et al. Preexposure chemoprophylaxis for HIV prevention in men who have sex with men. N Engl J Med. 2010;363:2587-99.

4. Thigpen MC, Kebaabetswe PM, Paxton LA, Smith DK, Rose CE, Segolodi TM, et al. Antiretroviral preexposure prophylaxis for heterosexual HIV transmission in Botswana. N Engl J Med. 2012;367:423-34.

5. US Food and Drug Administration. UFaD. FDA approves first drug for reducing the risk of sexually acquired HIV infection. 2012. http://www.fda.gov/NewsEvents/Newsroom/PressAnnouncements/ ucm312210.htm. Accessed 16 July 2012.

6. Molina JM, Capitant C, Spire B, Pialoux G, Chidac C, Charreau I, et al. On demand PrEP with oral TDF-FTC in MSM: results of the ANRS Ipergay trial. In: Conference on retroviruses and opportunistic infections, Seattle, WA, Feb 23-26, 2015.

7. McCormack S, Dunn D. Pragmatic open-label randomised trial of preexposure prophylaxis: the PROUD study. In: Conference on retroviruses and opportunistic infections, Seattle, WA, Feb 23-26, 2015.

8. Van Damme L, Corneli A, Ahmed K, Agot K, Lombaard J, Kapiga S, et al. Preexposure prophylaxis for HIV infection among African women. N Engl J Med. 2012;367:411-22.

9. Marrazzo JM, Ramjee G, Richardson BA, Gomez K, Mgodi N, Nair G, et al. Tenofovir-based preexposure prophylaxis for HIV infection among African women. N Engl J Med. 2015;372: 509-18.

10. Hendrix $\mathrm{CW}$. The clinical pharmacology of antiretrovirals for HIV prevention. Curr Opin HIV AIDS. 2012;7:498-504.

11. Amico KR. Adherence to preexposure chemoprophylaxis: the behavioral bridge from efficacy to effectiveness. Curr Opin HIV AIDS. 2012;7:542-8.

12. Corneli A, Perry B, Agot K, Ahmed K, Malamatsho F, Van Damme L. Facilitators of adherence to the study pill in the FEMPrEP clinical trial. PLoS One. 2015;10:e0125458.
13. Corneli AL, McKenna K, Perry B, Ahmed K, Agot K, Malamatsho F, et al. The science of being a study participant: FEMPrEP participants' explanations for overreporting adherence to the study pills and for the whereabouts of unused pills. J Acquir Immune Defic Syndr. 2015;68:578-84.

14. van der Straten A, Stadler J, Luecke E, Laborde N, Hartmann M, Montgomery ET. Perspectives on use of oral and vaginal antiretrovirals for HIV prevention: the VOICE-C qualitative study in Johannesburg, South Africa. J Int AIDS Soc. 2014; $17: 19146$.

15. van der Straten A, Stadler J, Montgomery E, Hartmann M, Magazi B, Mathebula F, et al. Women's experiences with oral and vaginal pre-exposure prophylaxis: the VOICE-C qualitative study in Johannesburg, South Africa. PLoS One. 2014;9: e89118.

16. Bekker LG, Hughes JP, Amico KR, Roux S, Hendrix C, Anderson PL, et al. HPTN 067/ADAPT Cape Town: a comparison of daily and nondaily PrEP dosing in African women. In: Conference on retroviruses and opportunistic infections, Seattle, WA, Feb 23-26, 2015.

17. Amico KR, McMahan V, Goicochea P, Vargas L, Marcus JL, Grant RM, et al. Supporting study product use and accuracy in self-report in the iPrEx study: next step counseling and neutral assessment. AIDS Behav. 2012;16:1243-59.

18. van der Straten A, Montgomery ET, Musara P, Etima J, Naidoo $\mathrm{S}$, Laborde N, et al. Disclosure of pharmacokinetic drug results to understand nonadherence. AIDS. 2015;29:2161-71.

19. Corneli AL, McKenna K, Headley J, Ahmed K, Odhiambo J, Skhosana $\mathbf{J}$, et al. A descriptive analysis of perceptions of HIV risk and worry about acquiring HIV among FEM-PrEP participants who seroconverted in Bondo, Kenya, and Pretoria, South Africa. J Int AIDS Soc. 2014;17:19152.

20. Bronfenbrenner U. The ecology of human development. US: President and Fellows of Havard College; 1979.

21. Eagly AH, Chaiken S. The advantages of an inclusive definition of attitude. Soc Cogn. 2007;25:582-602.

22. Sofolahan-Oladeinde Y, Mullins CD, Baquet CR. Using community-based participatory research in patient-centered outcomes research to address health disparities in under-represented communities. J Comp Eff Res. 2015;4:515-23.

23. Malterud K, Siersma VD, Guassora AD. Sample size in qualitative interview studies: guided by information power. Qual Health Res. 2015.

24. Fisher JD, Fisher WA, Amico KR, Harman JJ. An informationmotivation-behavioral skills model of adherence to antiretroviral therapy. Health Psychol. 2006;25:462-73.

25. Mays N, Pope C. Qualitative research: observational methods in health care settings. BMJ. 1995;311:182-4.

26. Rictchie J, Lewis J. Qualitative research practice: a guide for social science students and researchers. London: Sage; 2003.

27. Pope C, Ziebland S, Mays N. Qualitative research in health care. Analysing qualitative data. BMJ. 2000;320:114-6.

28. Campbell JL, Quincy C, Osserman J, Pedersen OK. Coding indepth semistructured interviews: problems of unitization and intercoder reliability and agreement. Sociol Methods \& Res. 2013;42:294-320.

29. Armstrong D, Gosling A, Weinman J, Marteau T. The place of inter-rater reliability in qualitative research: an empirical study. Sociol- J Br Sociol Assoc. 1997;31:597-606.

30. Langebeek N, Gisolf EH, Reiss P, Vervoort SC, Hafsteinsdottir TB, Richter C, et al. Predictors and correlates of adherence to combination antiretroviral therapy (ART) for chronic HIV infection: a meta-analysis. BMC Med. 2014;12:142.

31. Molloy GJ, Graham H, McGuinness H. Adherence to the oral contraceptive pill: a cross-sectional survey of modifiable behavioural determinants. BMC Public Health. 2012;12:838. 
32. Van der Elst EM, Mbogua J, Operario D, Mutua G, Kuo C, Mugo $\mathrm{P}$, et al. High acceptability of HIV pre-exposure prophylaxis but challenges in adherence and use: qualitative insights from a phase I trial of intermittent and daily PrEP in at-risk populations in Kenya. AIDS Behav. 2012.

33. Viswanathan M, Ammerman A, Eng E, Garlehner G, Lohr KN, Griffith D, et al. Community-based participatory research: assessing the evidence. Evid Rep Technol Assess (Summ). 2004; $1-8$.

34. UNAIDS. Good participatory practical guildelines for biomedical HIV prevention trials. Joint United Nations Programme on HIV/ AIDS 2011.

35. Shelton RC, Winkel G, Davis SN, Roberts N, Valdimarsdottir H, Hall SJ, et al. Validation of the group-based medical mistrust scale among urban black men. J Gen Intern Med. 2010;25:549-55.

36. Thompson HS, Valdimarsdottir HB, Winkel G, Jandorf L, Redd $\mathrm{W}$. The group-based medical mistrust scale: psychometric properties and association with breast cancer screening. Prev Med. 2004;38:209-18.

37. Rogers E. Diffusion of innovations. 5th ed. New York: Simon and Schister, Inc; 2003.
38. Prochaska JO, DiClemente CC. Stages of change in the modification of problem behaviors. Prog Behav Modif. 1992;28:183-218.

39. Tritter JQ, McCallum A. The snakes and ladders of user involvement: moving beyond Arnstein. Health Policy. 2006;76:156-68.

40. Barsdorf NW, Wassenaar DR. Racial differences in public perceptions of voluntariness of medical research participants in South Africa. Soc Sci Med. 2005;60:1087-98.

41. Earnshaw VA, Bogart LM, Dovidio JF, Williams DR. Stigma and racial/ethnic HIV disparities: moving toward resilience. Am Psychol. 2013;68:225-36.

42. Eaton LA, Driffin DD, Kegler C, Smith H, Conway-Washington $\mathrm{C}$, White $\mathrm{D}$, et al. The role of stigma and medical mistrust in the routine health care engagement of black men who have sex with men. Am J Public Health. 2015;105:e75-82.

43. Penner LA, Hagiwara N, Eggly S, Gaertner SL, Albrecht TL, Dovidio JF. Racial healthcare disparities: a social psychological analysis. Eur Rev Soc Psychol. 2013;24:70-122.

44. Cooper LA, Hill MN, Powe NR. Designing and evaluating interventions to eliminate racial and ethnic disparities in health care. J Gen Intern Med. 2002;17:477-86. 